\title{
Synthesis of Uniform Amplitude Focused Beam Arrays
}

\author{
Benjamin Fuchs, Member, IEEE, Anja Skrivervik, and Juan R. Mosig, Fellow, IEEE
}

\begin{abstract}
An iterative procedure for the synthesis of uniform amplitude focused beam arrays is presented. Specifically, the goal is to optimize the locations of a fixed number of array elements with known excitations in order to synthesize narrow-beam low-sidelobe patterns. Any fixed elements excitations can be handled, although uniform amplitude and equiphase excitations are aimed because of their practical interest. Moreover, the method can be applied to both linear and planar arrays, and there is no restriction regarding the element patterns. On top of being easy to implement, the proposed procedure leads to solutions that either equal or outperform the ones found by previous approaches in various cases of interest.
\end{abstract}

Index Terms-Antenna arrays, antenna synthesis, convex optimization.

\section{INTRODUCTION}

$\mathbf{T}$ O DESIGN antenna arrays that are attractive in terms of cost, reliability, and power efficiency, it is important to minimize the number of control points, i.e., to reduce as much as possible the required number of amplifiers and phase shifters. This trend is especially relevant in the area of satellite applications, where the complexity of the beamforming network is a key issue as highlighted in [1], [2].

In order to simplify the array implementation, the typical excitation tapering used to shape the beam can be replaced by properly choosing the locations of equally excited radiating elements. Although the synthesis of nonuniformly spaced arrays with fixed excitations has received a lot of attention these last 50 years [3]-[5], this problem remains challenging. The far field radiated by the array does indeed not depend linearly on the unknowns of the problem (the element locations), which makes the synthesis problem difficult to solve in an optimal way.

Only the most recent and relevant synthesis methods are reviewed here before introducing the proposed approach. Many global optimization algorithms have been used to circumvent the nonconvexity inherent to the synthesis of nonuniformly spaced arrays with known excitations [6]-[8]. However, it is

Manuscript received June 08, 2012; revised August 07, 2012; accepted September 25, 2012. Date of publication October 02, 2012; date of current version nulldate.

B. Fuchs is with the Laboratory of Electromagnetics and Acoustics (LEMA), Ecole Polytechnique Fédérale de Lausanne (EPFL), 1015 Lausanne, Switzerland, on leave from CNRS, LEMA-EPFL, 1015 Lausanne, Switzerland (e-mail: b.fuchs@epfl.ch).

A. Skrivervik and J. R. Mosig are with the Laboratory of Electromagnetics and Acoustics (LEMA), Ecole Polytechnique Fédérale de Lausanne (EPFL), 1015 Lausanne, Switzerland (e-mail: anja.skrivervik@epfl.ch; juan.mosig@epfl.ch).

Color versions of one or more of the figures in this letter are available online at http://ieeexplore.ieee.org.

Digital Object Identifier 10.1109/LAWP.2012.2221452 well known that their computational burden increases rapidly with the number of unknowns (i.e., the number of array elements) and the optimality of the solution is not ensured. To overcome this drawback, a deterministic synthesis procedure based on the density taper strategy introduced by [5] has recently been proposed in [9] to synthesize uniform amplitude arrays. This method requires the a priori knowledge of a reference (if possible optimal) continuous or discrete source that is then cleverly discretized into an array of the sought-after type. On top of being computationally effective, this technique outperforms many approaches using so-called global optimizers. This work has recently been extended to synthesize phase-only reconfigurable isophoric arrays [10].

In this letter, an iterative approach is proposed to synthesize uniform amplitude focused beam arrays. It can be applied to either linear or planar arrays. Specifically, the goal is to optimize the locations of a fixed number of array elements with known excitations in order to generate a pattern having the lowest possible sidelobes for a given beamwidth. An iterative algorithm that consists in solving a sequence of convex optimization problems is proposed to achieve this purpose. The proposed strategy turns out to be very effective as illustrated by several numerical comparisons to existing approaches. Let us recall that convex optimization methods were first introduced by [11] to efficiently solve array synthesis.

This letter is organized as follows. In Section II, the uniform amplitude array synthesis problem is described, and the proposed resolution procedure is detailed. To assess the capabilities of the method, numerical comparisons to other approaches are shown in Section III. Conclusions are drawn in Section IV.

\section{Problem Formulation AND ReSOlution}

\section{A. Antenna Array Synthesis Problem}

Let us consider an array composed of $N$ elements. Each element radiates a pattern $g_{n}(\theta, \varphi)$ in the direction $(\theta, \varphi)$ and is fed by a complex excitation $w_{n}$, with $n=1, \cdots, N$. The far fields $f(\theta, \varphi)$ radiated by linear and planar arrays are then respectively equal to

$$
\sum_{n=1}^{N} g_{n}(\theta, \varphi) w_{n} e^{j u x_{n}} \quad \text { and } \quad \sum_{n=1}^{N} g_{n}(\theta, \varphi) w_{n} e^{j\left(u x_{n}+v y_{n}\right)}
$$

with $u=2 \pi \sin \theta \cos \varphi, v=2 \pi \sin \theta \sin \varphi$ and where $x_{n}$ and $y_{n}$ are the element locations in wavelengths. The schematic view of a planar array with the associated notations is represented in Fig. 1.

In the synthesis problem, the element patterns $g_{n}(\theta, \varphi)$ and the excitations $w_{n}$ are fixed, whereas the element locations 


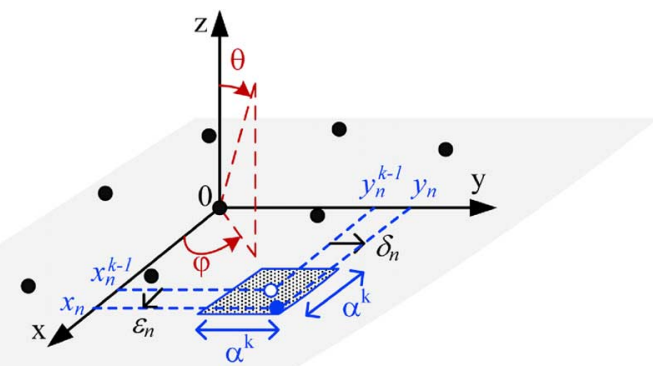

Fig. 1. Schematic view of the planar array with the notations and coordinate system.

$\left(x_{n}, y_{n}\right)$ are left free. In practice, a specific, very interesting case is the synthesis of so-called uniform amplitude arrays, i.e., arrays with excitations of the same magnitude and phase: $\left|w_{n}\right|=$ $A$ and $\angle w_{n}=\Phi_{0} \forall n$ where $A$ and $\Phi_{0}$ are arbitrary constants.

For the sake of clarity but without any loss of generality, one considers uniform amplitude arrays excited by $w_{n}=1 / N$ that are composed of isotropic elements $g_{n}(\theta, \varphi)=1$. The far fields $f(\theta, \varphi)$ in (1) become

$$
\frac{1}{N} \sum_{n=1}^{N} e^{j u x_{n}} \quad \text { and } \quad \frac{1}{N} \sum_{n=1}^{N} e^{j\left(u x_{n}+v y_{n}\right)}
$$

for linear and planar arrays, respectively. The sidelobe region $S$ where the magnitude of the field is upper-bounded by an envelope $e(\theta, \varphi)$ is introduced. This envelope is considered equal to a constant $\rho$ in the following. The complementary domain of $S$ defines the main beam region and therefore the main beamwidth. Since the magnitude of the field is equal to one toward broadside, $|f(\theta=0, \varphi)|=1$ [see (2)], the synthesis problem amounts to minimize $\rho$ over $S$. It can be written

$$
\min _{\boldsymbol{x}, \boldsymbol{y}} \rho, \quad \text { under } \quad \sup _{(\theta, \varphi) \in S}|f(\theta, \varphi)| \leq \rho
$$

where $\boldsymbol{x}=\left(x_{1}, \ldots, x_{N}\right)$ and $\boldsymbol{y}=\left(y_{1}, \ldots, y_{N}\right)$ are the $N$-dimensional element location vectors to be determined. This optimization problem is not convex and difficult to solve in an optimal way.

\section{B. Resolution Method}

An iterative algorithm is proposed to solve the synthesis problem (3). It consists in solving a sequence of convex optimization problems. Let us first present the convex problem addressed at iteration $k$ before describing the iterative algorithm.

1) Convex Optimization Problem at Iteration $k$ : The far field $f(\theta, \varphi)$ radiated by the array (2) does not depend linearly on the variables $(\boldsymbol{x}, \boldsymbol{y})$. To overcome this problem, one linearizes (2) around the current (suboptimal) solution, i.e., the element locations $\left(\boldsymbol{x}^{k-1}, \boldsymbol{y}^{k-1}\right)$. For this purpose, let us define

$x_{n}=x_{n}^{k-1}+\epsilon_{n} \quad$ and $\quad y_{n}=y_{n}^{k-1}+\delta_{n}, \quad$ for $n=1, \ldots, N$

where $\epsilon_{n}$ and $\delta_{n}$ are the location displacements of the element $n$ according to the $x$ - and $y$-axis, respectively, as depicted in Fig. 1.

Using the Taylor expansion $e^{j \gamma} \approx 1+j \gamma$, the far fields (2) can be approximated at iteration $k$ by

$$
f_{\epsilon_{n}}^{k}(\theta, \varphi)=\frac{1}{N} \sum_{n=1}^{N} e^{j u x_{n}^{k-1}}\left(1+j u \epsilon_{n}\right)
$$

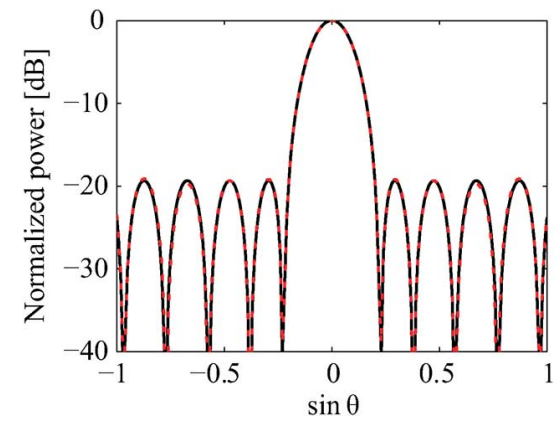

Fig. 2. Synthesized far fields using the proposed procedure (solid line) and the deterministic method [9] of a 10-element uniform amplitude array.

TABLE I

OPTIMIZED LOCATIONS OF THE 10-ELEMENT LINEAR ARRAY

\begin{tabular}{ccc|ccc}
\hline \hline$n$ & $x_{n} / \lambda$ & $x_{n} / \lambda$ of [9] & $n$ & $x_{n} / \lambda$ & $x_{n} / \lambda$ of [9] \\
\hline 1 & -2.2500 & -2.2500 & 6 & 0.2020 & 0.2019 \\
2 & -1.6024 & -1.6026 & 7 & 0.6156 & 0.6158 \\
3 & -1.0612 & -1.0671 & 8 & 1.0612 & 1.0671 \\
4 & -0.6156 & -0.6158 & 9 & 1.6024 & 1.6026 \\
5 & -0.2020 & -0.2019 & 10 & 2.2500 & 2.2500 \\
\hline \hline
\end{tabular}

and

$$
f_{\epsilon_{n}, \delta_{n}}^{k}(\theta, \varphi)=\frac{1}{N} \sum_{n=1}^{N} e^{j\left(u x_{n}^{k-1}+v y_{n}^{k-1}\right)}\left(1+j u \epsilon_{n}+j v \delta_{n}\right) .
$$

In general, $f_{\epsilon_{n}, \delta_{n}}^{k}(\theta, \varphi)$ is a good approximation of $f(\theta, \varphi)$ in (2) provided that $\left|u \epsilon_{n}\right| \ll 1$ and $\left|v \delta_{n}\right| \ll 1$, i.e., that $\left|\epsilon_{n}\right|$ and $\left|\delta_{n}\right|$ are much smaller than $1 /(2 \pi) \approx 0.16$. In practice, $(5)$ may be valid for larger values of $\left|\epsilon_{n}\right|$ and $\left|\delta_{n}\right|$ than expected.

By introducing the $N$-dimensional element displacement vectors $\boldsymbol{\epsilon}=\left(\epsilon_{1}, \ldots, \epsilon_{N}\right)$ and $\boldsymbol{\delta}=\left(\delta_{1}, \ldots, \delta_{N}\right)$, the problem solved at iteration $k$ of the algorithm is

$$
\min _{\boldsymbol{\epsilon}, \boldsymbol{\delta}} \rho, \quad \text { under }\left\{\begin{array}{l}
\sup _{(\theta, \varphi) \in S}\left|f_{\boldsymbol{\epsilon}, \boldsymbol{\delta}}^{k}(\theta, \varphi)\right| \leq \rho \\
\left|\epsilon_{n}\right| \leq \alpha^{k} \text { and }\left|\delta_{n}\right| \leq \alpha^{k}, \quad n=1, \ldots, N
\end{array}\right.
$$

where the upper bound $\alpha^{k}$ on the location displacements allows to control the degree of approximation of the far fields (5).

The optimization problem (6) is a nonlinear convex problem and specifically a second-order cone program [12]. It can thus be efficiently solved using interior point methods by many readily available solvers in roughly the same time as a linear program of equivalent size. Examples of second-order cone programs applied to various antenna array synthesis problems can be found in [12] and [13].

Note that for the numerical implementation, the constraint $\sup _{(\theta, \varphi) \in S}\left|f_{\boldsymbol{\epsilon}, \boldsymbol{\delta}}^{k}(\theta, \varphi)\right| \leq \rho$ in (6) is approximated by $(\theta, \varphi) \in S$

$$
\max _{\substack{i=\left\{1, \ldots, M_{\theta}\right\} \\ j=\left\{1, \ldots, M_{\varphi}\right\}}}\left|f_{\boldsymbol{\epsilon}, \boldsymbol{\delta}}^{k}\left(\theta_{i}, \varphi_{j}\right)\right| \leq \rho
$$

where $\left(\theta_{i}, \varphi_{j}\right)$ are $M_{\theta} \times M_{\varphi}$ sample points in the sidelobe region $S$. 


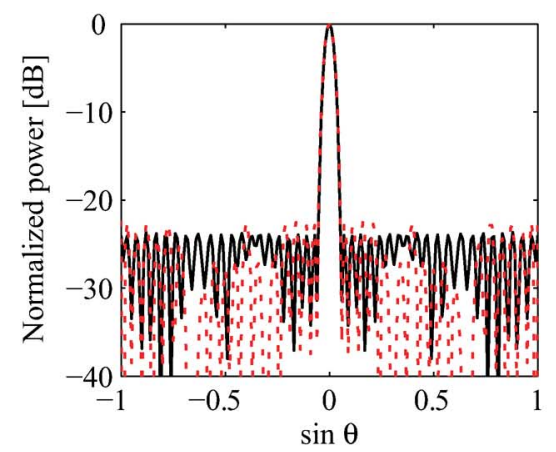

(a)

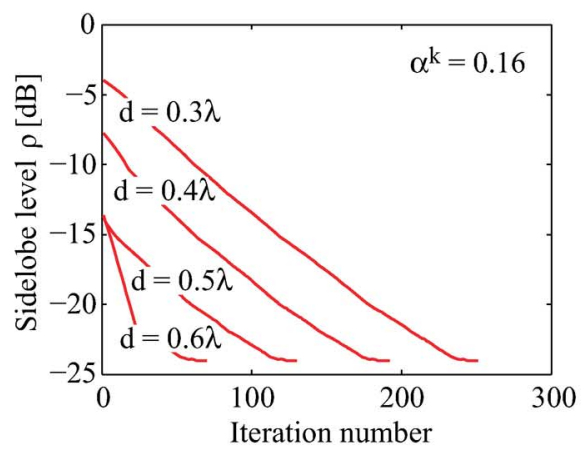

(b)

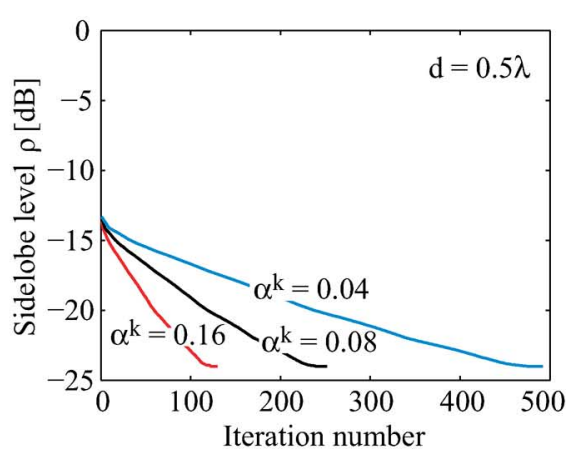

(c)

Fig. 3. Synthesis results of a 32-element uniform amplitude array: (a) far-field patterns synthesized via convex optimizations (solid line) and via differential evolution algorithm [6] (dashed line) with the convergence of the proposed iterative procedure, i.e., the sidelobe level as a function of the iteration number, for (b) various starting interelement spacings $d$ and (c) various upper bounds $\alpha^{k}$.

2) Iterative Algorithm: A sequence of convex optimization problems (6) is solved to synthesize uniform amplitude arrays. The iterative algorithm is as follows.

- In the first iteration $(k=1)$, one starts with an equispaced array. The initial element locations in (4) are such as: $x_{n}^{0}=$ $y_{n}^{0}=(n-1) d$, where $d$ is the interelement spacing in wavelengths. Unless specified otherwise, $d$ is equal to 0.5 .

1) For $k \geq 1$, solve the convex optimization problem (6) to get $\left(\epsilon_{n}^{k}, \delta_{n}^{k}\right)$ with typically $\alpha^{k}=0.16$, if $f_{\epsilon_{n}, \delta_{n}}^{k}(\theta, \varphi)$ in (5) is a good approximation of $f(\theta, \varphi)$ in (2).

2) Update the solution: $x_{n}^{k}=x_{n}^{k-1}+\epsilon_{n}^{k}$ and $y_{n}^{k}=y_{n}^{k-1}+\delta_{n}^{k}$.

3) Repeat steps 1 and 2 until $20 \log \left|\rho^{k+1} / \rho^{k}\right| \leq 0.01$, i.e., until the maximum sidelobe level no longer diminishes.

There is, of course, no guarantee that the final solution of the iterative algorithm is the optimal solution of the synthesis problem (3). The initial setting $d=0.5$ and $\alpha^{k}=0.16$ is used although another choice may lead to a faster convergence in some cases. However, it has been experimentally noticed that the convergence point is robust to the choice of $d$ and $\alpha^{k}$. Moreover, the synthesized uniform arrays compare favorably to the solutions found in the literature as shown in Section III.

Note that constraints on the maximum array dimensions for both linear and planar arrays can be fixed. Moreover, a minimum element spacing for linear arrays can be set while keeping the convexity of the problem.

\section{NUMERICAL APPLICATION-COMPARISONS TO OTHER APPROACHES}

Various numerical applications of the synthesis of uniform amplitude focused beam arrays are presented. Comparisons to results available in the literature first assess the validity of the proposed procedure. Then, the interest of uniform and stepped amplitude array is shown by comparison to an optimally excited array. Note that all simulations are carried out on a $2.93-\mathrm{GHz}$ CPU 8 Go-RAM computer.

\section{A. Comparisons to Deterministic Approach}

A deterministic approach has been presented in [9] to synthesize uniform amplitude arrays. This technique is used as a reference to assess the proposed method.

The goal of the deterministic approach is to optimize the 10 element locations of a uniform amplitude linear array in order to match a radiation pattern corresponding to a Taylor distribution with SLL $=-20 \mathrm{~dB}, a=2.47 \lambda$, and $\bar{n}=10$ [14]. The outermost elements have been fixed to $\pm 2.25 \lambda$. The synthesized pattern has a half-beamwidth $\theta_{s}$ such as $\sin \theta_{s}=0.2$, and the achieved sidelobe levels are below $-19.2 \mathrm{~dB}$.

The proposed approach is applied to synthesize an uniform amplitude array of the same length that radiates a focused beam having the same beamwidth. The proposed approach recovers the results found by the determinitic method [9] in less than $5 \mathrm{~s}$ : The synthesized far-field patterns are almost superimposed as shown in Fig. 2, and the optimized element locations given in Table I are very close.

\section{B. Comparison to Global Optimization Method}

The synthesis of a 32-element uniform amplitude array is now considered. A global optimization method (the differential evolution algorithm) is used in [6] to synthesize a narrow-beam low-sidelobe pattern. For a maximum interelement spacing of $1 \lambda$ and a $6^{\circ}$ beamwidth, a minimum sidelobe level of $-22.5 \mathrm{~dB}$ is achieved.

The proposed method is applied to synthesize a uniform amplitude array having the same beamwidth. A minimum sidelobe level of $-24.0 \mathrm{~dB}$ is reached for a slightly larger array in less than $3 \mathrm{~min}$. Similar performances (i.e., sidelobe level of $-22.5 \mathrm{~dB}$ ) are obtained for the same array length. The synthesized far-field patterns are plotted in Fig. 3(a) and compared to those obtained by [6]. The convergence of the proposed iterative algorithm, quantified by the sidelobe level as a function of the iteration number, is shown in Fig. 3(b) and (c). The convergence speed depends on the choice of the interelement spacings $d$ and the upper bound $\alpha_{k}$. However, the choice of these settings does not change the solution, i.e., the optimized element locations and therefore the achieved sidelobe level.

\section{Comparisons to Optimally Excited Equispaced Planar Array}

The synthesis of a $1 \lambda$ side planar array composed of $5 \times 5$ elements with pattern $g_{n}(\theta)=\cos \theta$ is addressed.

On the one hand, a $\lambda / 2$ equispaced planar array is considered. The excitations are optimally determined to radiate a pattern of beamwidth equal to $\sin \theta_{s}=0.45$, as described in [13]. A maximum sidelobe level of $-24.3 \mathrm{~dB}$ is achieved, and the ratio 


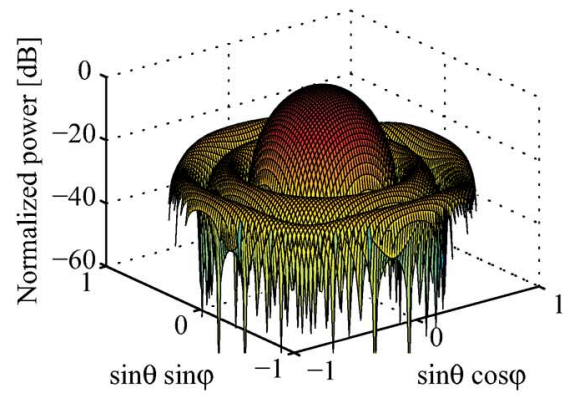

(a)

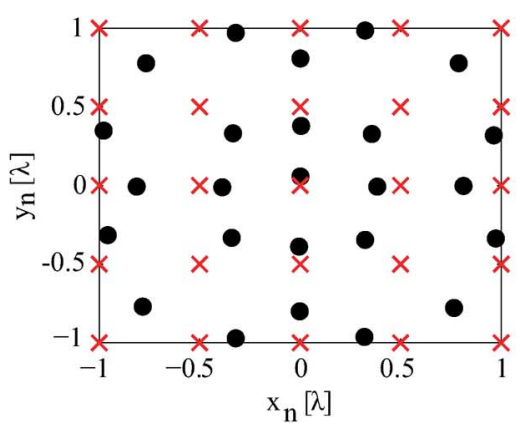

(b)

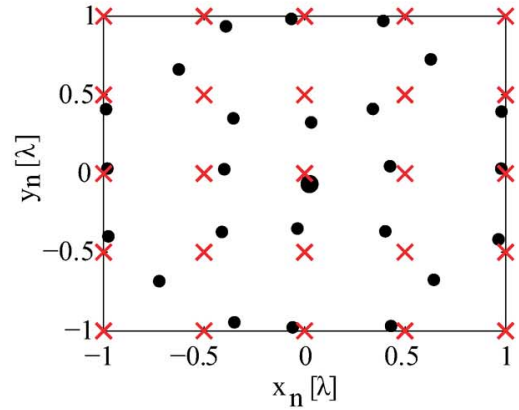

(c)

Fig. 4. Narrow-beam low-sidelobe synthesis of a $5 \times 5$-element planar array. (a) Far-field pattern synthesized by the optimally excited equispaced array and layout of (b) the uniform amplitude array (large $\bullet$ for $w_{n}=1$ ) and (c) the stepped amplitude array (large $\bullet$ for $w_{n}=1$ and small $\bullet$ for $w_{n}=0.5$ ) compared to the equispaced array $(x)$.

between the maximum and minimum weight magnitude is equal to 18.2 .

On the other hand, the element locations of both a uniformly excited array $\left(w_{n}=1, \forall n\right)$ and a stepped amplitude array $\left(w_{1}=1\right.$ and $w_{n}=0.5$, for $\left.n=2, \ldots, 25\right)$ are optimized using the proposed procedure.

For the same beamwidth $\sin \theta_{s}=0.45$, a maximum sidelobe level of $-25.2 \mathrm{~dB}$ and of $-26.0 \mathrm{~dB}$ for the uniformly excited array and the stepped amplitude array, respectively, is reached in less than $5 \mathrm{~min}$. It means that better performances can be achieved for the same maximum array dimension by optimizing the element locations instead of the element excitations. The far-field patterns and array layouts for both uniform and stepped amplitude arrays are plotted in Fig. 4.

\section{CONCLUSION}

An iterative method to synthesize uniform amplitude focused beam arrays has been presented. Each iteration of the algorithm simply requires solving a convex optimization problem, which means that many readily available routines can be used to efficiently solve the problem. On top of being easy to implement and computationally effective, the method can be applied to synthesize both linear and planar arrays, and there is no restriction regarding the element patterns.

While the optimality of the solution cannot be guaranteed, the performances of the synthesized designs compare favorably to solutions found by previously proposed approaches. Numerical comparisons to a global optimization based method and a deterministic technique indeed show the validity of the obtained results. Finally, a planar array composed of nonisotropic elements with uniform and stepped amplitudes is synthesized to both highlight the potentialities of the method and show the interest of designing such arrays.

Note that the choice of an initial equispaced array may not always be the best one. For some particular cases, the density taper procedure could, for instance, be used to compute a better starting guess.

\section{REFERENCES}

[1] G. Caille, Y. Cailloce, C. Guiraud, D. Auroux, T. Touya, and M. Masmousdi, "Large multibeam array antennas with reduced number of active chains," in Proc. Eur. Conf. Antennas Propag., Edinburgh, U.K., Nov. 2007, pp. 1-9.

[2] M. C. Viganó, G. Toso, G. Caille, C. Mangenot, and I. E. Lager, "Spatial density tapered sunflower antenna array," in Proc. Eur. Conf. Antennas Propag., Berlin, Germany, Mar. 2009, pp. 778-782.

[3] R. E. Willey, "Space tapering of linear and planar arrays," IRE Trans. Antennas Propag., vol. AP-10, no. 4, pp. 369-377, Jul. 1962.

[4] Y. T. Lo, "A study of spaced-tapered arrays," IEEE Trans. Antennas Propag., vol. 14, no. 1, pp. 22-30, Jan. 1966.

[5] M. I. Skolnik, "Nonuniform arrays," in Antenna Theory, R. E. Collin and F. J. Zucker, Eds. New York: McGraw-Hill, 1969, ch. 6, pt. 1.

[6] D. G. Kurup, M. Himdi, and A. Rydberg, "Synthesis of uniform amplitude unequally spaced antenna arrays using the differential evolution algorithm," IEEE Trans. Antennas Propag., vol. 51, no. 9, pp. 2210-2217, Sep. 2003.

[7] N. Jin and Y. Rahmat-Samii, “Advances in particle swarm optimization for antenna designs: Real-number, binary, single-objective and multiobjective implementations," IEEE Trans. Antennas Propag., vol. 55, no. 3, pp. 556-567, Mar. 2007.

[8] H. Oraizi and M. Fallahpour, "Nonuniformly spaced linear array design for the specified beamwidth/sidelobe level or specified directivity/sidelobe level with coupling considerations," Prog. Electromagn. Res. M, vol. 4, pp. 185-209, 2008.

[9] O. M. Bucci, M. D’Urso, T. Isernia, P. Angeletti, and G. Toso, “Deterministic synthesis of uniform amplitude sparse arrays via new density taper techniques," IEEE Trans. Antennas Propag., vol. 58, no. 6, pp. 1949-1958, Jun. 2010.

[10] A. F. Morabito, T. Isernia, and L. Di Donato, "Optimal synthesis of phase-only reconfigurable linear sparse arrays having uniform-amplitude excitations," Prog. Electromagn. Res., vol. 124, pp. 405-423, 2012.

[11] H. Lebret and S. Boyd, "Antenna pattern synthesis via convex optimization," IEEE Trans. Signal Process., vol. 45, no. 3, pp. 526-531, Mar. 1997.

[12] M. Lobo, L. Vandenberghe, S. Boyd, and H. Lebret, "Applications of second-order cone programming," Lin. Algebra Appl., vol. 284, no. 1-3, pp. 193-228, 1998.

[13] B. Fuchs and J. J. Fuchs, "Optimal narrow beam low sidelobe synthesis for arbitrary arrays," IEEE Trans. Antennas Propag., vol. 58, no. 6, pp. 2130-2135, Jun. 2010.

[14] T. T. Taylor, "Design of line-source antennas for barrow beamwidth and low sidelobes," IRE Trans. Antennas Propag., vol. AP-3, no. 1, pp. 16-28, Jan. 1955. 